\section{Red Regional de Laboratorios para la Vigilancia de las Infecciones Fúngicas Invasoras y Susceptibilidad a los Antifúngicos}

\author{
Manuel Cuenca-Estrella, ${ }^{1}$ \\ Juan L. Rodríguez-Tudela, ${ }^{1}$ \\ Susana Córdoba, ${ }^{2}$ Marcia C. Melhem, ${ }^{3}$ \\ María W. Szeszs, ${ }^{3}$ \\ Elizabeth Castañeda, ${ }^{4}$ \\ Gerardo Martínez ${ }^{5}$ \\ y Jean-Marc Gabastou ${ }^{6}$
}

Forma de citar: Cuenca-Estrella M, Rodríguez-Tudela JL, Córdoba S, Melhem MC, Szeszs MW, Castañeda E, et al. Red Regional de Laboratorios para la Vigilancia de las Infecciones Fúngicas Invasoras y Susceptibilidad a los Antifúngicos. Rev Panam Salud Publica. 2008;23(2):129-34.

Palabras clave: micosis, vigilancia, diagnóstico, antifúngicos, Latinoamérica, Organización Panamericana de la Salud.

\footnotetext{
1 Servicio de Micología, Centro Nacional de Microbiología, Instituto de Salud Carlos III, Majadahonda, España. La correspondencia se debe dirigir al Dr. Manuel Cuenca-Estrella. Director Adjunto, Servicio de Micología, Centro Nacional de Microbiología, Instituto de Salud Carlos III. Carretera Majadahonda-Pozuelo Km 2. 28220 Majadahonda (Madrid), España. Tel.: + 34-91-5097961. Fax: + 34-915097966. Correo electrónico : mcuenca-estrella@isciii.es

2 Departamento de Micología, Instituto Nacional de Enfermedades Infecciosas, ANLIS, Malbrán, Buenos Aires, Argentina.

3 Seção de Micología. Serviço de Parasitología, Instituto Adolfo Lutz, Sao Paulo, Brasil.

4 Grupo de Microbiología, Instituto Nacional de Salud, Bogotá, Colombia.

5 Departamento de Bacteriología/Micología del Instituto de Medicina Tropical "Pedro Kourí", La Habana, Cuba.

6 Unidad de Medicamentos Esenciales, Vacunas y Tecnología de la Salud, Organización Panamericana de la Salud, Quito, Ecuador.
}

\section{OBJETIVOS DE LA RED}

Las infecciones fúngicas han aumentado en frecuencia e importancia en los últimos años (1-3). La introducción de los antibióticos en 1945, de la corticoterapia en 1959 y del tratamiento con inmunosupresores en 1960, provocó un aumento, sin precedentes, de la incidencia de las infecciones fúngicas en todo el mundo. Estos avances médicos han permitido mejorar el pronóstico de muchas enfermedades aunque, paralelamente, han dado lugar a un incremento de las complicaciones iatrogénicas y de las infecciones oportunistas, entre las que se encuentran las micosis. A partir de1970, la incidencia anual de las infecciones fúngicas ha aumentado de 3 a 20 veces, dependiendo del tipo de infección y del trabajo consultado (4-13).

Por otra parte, con la aparición del VIH/SIDA al principio de la década de 1980, la población susceptible de padecer infecciones oportunistas (IO) se incrementó de manera considerable. En sus inicios, por sólo citar algunos ejemplos, ciertas micosis invasoras, como la neumocistosis, afectaron de un $70 \%$ a un $80 \%$ de la población con SIDA, con una mortalidad asociada de $20 \%$ a $40 \%$. Por otro lado, la criptococosis pasó de $5 \%$ a $8 \%$ en países desarrollados, y a más de $20 \%$ en regiones de África, con una tasa de mortalidad superior a $30 \%$; por su parte, la histoplasmosis ascendió de $2 \%$ a $5 \%$ en regiones endémicas (14). En las dos últimas décadas, como resultado de los tratamientos profilácticos y de la terapia antirretroviral de gran efectividad, la incidencia de las IO ha disminuido; sin embargo, sus tasas de morbilidad y mortalidad siguen siendo elevadas, en particular en países de bajos recursos (15).

Además, otros estudios epidemiológicos publicados en los últimos años han destacado que la elevada prevalencia de las infecciones fúngicas se acompaña de una mortalidad preocupante. De esta forma, la mortalidad atribuible a la candidemia se sitúa entre $20 \%$ y $50 \%$ de los casos; la correspondiente a la aspergilosis entre $40 \%$ y $80 \%$ de los casos $\mathrm{y}$, en algunas infecciones por hongos emergentes, la tasa de mortalidad puede ir más allá de 90\% (1619). Las cifras de mortalidad dependen de muchos factores concomitantes, como la enfermedad de base, la zona geográfica, el tipo de infección y el tratamiento aplicado; empero, en términos generales las infecciones fúngicas oportunistas se están convirtiendo en un problema sanitario de primer orden, en todo el mundo desarrollado (20). Otros 
trabajos, quizá más ilustrativos, han demostrado que Candida spp. causan $10 \%$ de las sepsis nosocomiales y que la candidemia prolonga en tres semanas la estancia hospitalaria, duplicando el riesgo de muerte del enfermo $(11,18,19,21)$. En todo el mundo se realizan más de 650000 trasplantes al año, con un incremento anual medio de 1,5\%. La población de enfermos trasplantados constituye uno de los principales grupos de riesgo de micosis, ya que se calcula que de $10 \%$ a $15 \%$ de estos pacientes desarrollarán una infección fúngica. Esta cifra puede duplicarse en personas trasplantadas de precursores hematopoyéticos, para quienes se calcula una tasa de mortalidad atribuible a las micosis de $20 \%$ a $25 \%(17,22-24)$.

Esta nueva situación epidemiológica ha causado un incremento en el consumo de antifúngicos, así como el desarrollo de nuevas técnicas diagnósticas y terapéuticas. En la última década, el consumo de antifúngicos ha aumentado alrededor de $12 \%$ de media cada año, y según datos globales, el costo en antifúngicos asciende a 3600 millones de euros anuales $(25,26)$. El uso masivo de antifúngicos ha originado la aparición de resistencias secundarias y, quizá, el desplazamiento de especies sensibles por otras más resistentes $(27,28)$, por lo que se han desarrollado nuevas moléculas antifúngicas que ya se emplean en muchos hospitales. Ante esta nueva situación, es necesario conocer el perfil de susceptibilidad de las especies fúngicas para instaurar el tratamiento más adecuado, por lo que se han desarrollado métodos estandarizados para realizar estudios de susceptibilidad in vitro a los antifúngicos $(26,29)$.

Por tanto, el diagnóstico precoz, la caracterización de las especies y la detección de la resistencia a los antifúngicos se han situado como herramientas básicas para el control de las infecciones fúngicas. Sin embargo, estas técnicas no están aún implantadas en muchos laboratorios ya que son complejas y costosas y, por tanto, deben ser manejadas e interpretadas por personal experto.

Los laboratorios de referencia se han convertido en centros imprescindibles en la formación de personal, en la estandarización de técnicas microbiológicas, en la transferencia de tecnología y conocimiento a otros centros y en el desarrollo de programas de evaluación externa del desempeño. Asimismo, la interlocución y el intercambio de información entre estos centros son esenciales para conocer la auténtica realidad epidemiológica de las micosis $(3,1)$.

La Organización Panamericana de la Salud/ Organización Mundial de la Salud (OPS/OMS) recomienda fortalecer la capacidad de las redes de laboratorios e institutos de salud pública en América Latina y el Caribe, en una acción fundamental del quehacer colectivo de la comunidad sanitaria de las Américas, para el diagnóstico, la vigilancia, la prevención y el control de las enfermedades prevalentes, respondiendo a los nuevos desafíos epidemiológicos que se presentan en el hemisferio, dentro del marco de la aplicación del Reglamento Sanitario Internacional 2005.

En este contexto, en 2006, bajo la coordinación de la Unidad de Medicamentos Esenciales, Vacunas y Tecnologías en Salud de la OPS/OMS, con el apoyo técnico y financiero del Centro Nacional de Microbiología del Instituto de Salud Carlos III (ISC III), y el soporte técnico del Departamento de Micología del Instituto Nacional de Enfermedades Infecciosas, ANLIS, Dr. C. Malbrán, de Buenos Aires, Argentina y de la Seção de Micologia del Serviço de Parasitología, Instituto Adolfo Lutz, de São Paulo, Brasil, se creó la Red Regional de Vigilancia de las Infecciones Fúngicas Invasoras y Susceptibilidad a los Antifúngicos. Esta última se organizó a través del Plan de Acción Conjunta entre la Agencia Española de Cooperación Internacional (AECI) y la OPS, por medio del financiamiento concedido por el ISC III del Ministerio de Sanidad y Consumo de España.

Los objetivos de la Red recogen los aspectos expuestos en los párrafos anteriores y pueden enumerarse como: 1) la vigilancia epidemiológica de la infección fúngica invasora mediante la detección de resistencias a los antifúngicos y la caracterización de especies emergentes; 2) el establecimiento de normas y protocolos comunes para el diagnóstico precoz de las micosis y la detección de resistencias a los antifúngicos; 3) el fortalecimiento de los mecanismos de coordinación, comunicación y transferencia entre los países; 4) la diseminación de información epidemiológica veraz y confiable; 5) la capacitación técnica de profesionales mediante cursos, talleres y estadías en los laboratorios de la red; 6) el desarrollo de programas conjuntos de investigación y de innovación tecnológica, y 7) la implementación de un programa de evaluación externa del desempeño.

La Red está organizada en cuatro niveles de complejidad y se distribuye de la siguiente manera: 1) el Servicio de Micología del Centro Nacional de Microbiología, del Instituto de Salud Carlos III en Majadahonda, España, funge como centro regional de referencia para la Red Latinoamericana. 2) El Departamento de Micología del Instituto Nacional de Enfermedades Infecciosas Dr. C. Malbrán, en Buenos Aires, Argentina, y el Departamento de Micología y Parasitología del Instituto Adolfo Lutz, en São Paulo, Brasil, constituyen los dos centros de referencia sub-regionales. 3) Desde 2005, el Grupo de Microbiología del Instituto Nacional de Salud, en Bogotá, Colombia, y desde finales de 2006, el Departamento de Bacteriología/Micología del Insti- 
tuto de Medicina Tropical, Pedro Kourí (IPK) de La Habana, Cuba, se constituyeron en los dos centros nacionales de referencia. 4) En Argentina existe un centro centinela, al igual que 11 en Brasil, tres en Colombia y dos en Cuba, todos capacitados por los centros nacionales correspondientes entre 2002 y 2006.

El plan de acción de la Red consiste en la implantación de las técnicas de vigilancia en los laboratorios nacionales de referencia, para su posterior difusión por los centros hospitalarios de cada país. El desarrollo de las técnicas en los laboratorios de referencia se logra mediante un proceso gradual de transferencia tecnológica in situ, así como a través de la capacitación de los profesionales en cursos y talleres. Posteriormente, cada centro de referencia realiza un plan de acción similar en su zona de influencia, además de llevar a cabo la supervisión de las actividades de los centros hospitalarios en los que se implantan las técnicas de vigilancia.

La evaluación externa del desempeño de los centros de la Red Regional forma parte de las actividades de supervisión, así como de los programas para garantizar la calidad que debe regir en la obtención de información precisa y confiable. En el segundo semestre de 2006 se llevó a cabo un ejercicio de evaluación de los centros de referencia de la Red, mediante la realización de estudios de susceptibilidad a los antifúngicos con una colección de cepas control.

\section{LOGROS ALCANZADOS}

\section{Estudios de susceptibilidad}

Los laboratorios sub-regionales realizaron los estudios de susceptibilidad siguiendo estrictamente uno de los dos métodos de referencia recomendados para la Región, como el recogido en el documento M27-A2 del Clinical Laboratory Standards Institute (CLSI) estadounidense o el método del Antifungal Subcommittee of the European Committee on Antifungal Susceptibility Testing (AFSTEUCAST, document 7.1) (29, 30). Algunos de los laboratorios también realizaron las pruebas de susceptibilidad por un método de difusión o por un método comercial como el Etest (AB Biodisk, Solna, Sweden). En esos casos, se siguieron las recomendaciones del fabricante y los protocolos estandarizados de las técnicas de difusión (documento M44A del CLSI), tanto en la realización de las técnicas como en la interpretación de los resultados (31).

\section{Programa de evaluación externa del desempeño}

El centro de referencia regional del Instituto de Salud Carlos III, en su calidad de evaluador externo, envió una colección de ocho cepas de referencia a cada uno de los centros sub-regionales y nacionales de referencia. Seis de las cepas estaban codificadas sin que se supiera su especie ni su perfil de susceptibilidad y fueron elegidas por las características específicas de sensibilidad o resistencia a los antifúngicos. Las otras dos cepas, Candida krusei ATCC6258 y Candida parapsilosis ATCC22019 sirvieron de control interno para que los laboratorios evaluados validaran sus propios resultados antes de enviarlos al laboratorio evaluador externo. En los cuadros 1 y 2 se incluyen las identificaciones de las cepas analizadas y los intervalos de sensibilidad y resistencia a los antifúngicos por los métodos del EUCAST y de CLSI.

La evaluación externa se realizó calculando el porcentaje de concentraciones mínimas inhibitorias

CUADRO 1. Intervalos para validar los resultados obtenidos por el método del EUCASTa

\begin{tabular}{|c|c|c|c|c|c|c|c|c|}
\hline Cepa & Identificación & $\mathrm{ANB}^{\mathrm{b}}$ & $5-\mathrm{FC}^{\mathrm{C}}$ & $\mathrm{FLC}^{\mathrm{d}}$ & $\mathrm{ITC}^{\mathrm{e}}$ & $\operatorname{VRC}^{f}$ & KTCg & $\mathrm{CPF}^{\mathrm{h}}$ \\
\hline Cepa A, & Candida krusei ATCCi 6258 & $0,12-0,5$ & $2,0-8,0$ & $16,0-\geq 64,0$ & $0,03-0,25$ & $0,06-0,25$ & $0,06-0,25$ & $0,12-0,5$ \\
\hline Cepa B & Candida albicans ATCC 64548 & $0,03-0,25$ & $0,12-0,5$ & $0,12-0,5$ & $0,015-0,06$ & $0,015-0,03$ & $0,015-0,03$ & $0,03-0,12$ \\
\hline Cepa C & Candida albicans ATCC 64550 & $0,06-0,50$ & $0,5-4,0$ & $8,0-\geq 64,0$ & $0,25-1,0$ & $0,12-0,5$ & $0,12-0,5$ & $0,03-0,12$ \\
\hline Cepa D & Candida tropicalis ATCC 200956 & $1,0-8,0$ & $0,12-0,5$ & $32,0-\geq 64,0$ & $4,0-\geq 8,0$ & $\geq 8,0$ & $4,0-\geq 8,0$ & $0,03-0,25$ \\
\hline Cepa E & Candida glabrata ATCC 90030 & $0,06-0,50$ & $0,12-0,5$ & $2,0-16,0$ & $0,06-0,5$ & $0,06-0,25$ & $0,12-0,5$ & $0,12-1,0$ \\
\hline Cepa F & Saccharomyces cerevisiae ATCC 9763 & $0,06-0,50$ & $0,12-0,5$ & $2,0-8,0$ & $0,12-0,50$ & $0,015-0,12$ & $0,015-0,12$ & $0,25-1,0$ \\
\hline Cepa G & Candida Iusitaniae ATCC 200951 & $0,25-2,0$ & $0,12-0,5$ & $0,06-0,25$ & $0,015-0,03$ & $0,015-0,03$ & $0,015-0,03$ & $0,5-4,0$ \\
\hline Сера H & Candida parapsilosis ATCC 22019 & $0,12-0,5$ & $0,12-0,5$ & $0,5-2,0$ & $0,03-0,12$ & $0,015-0,06$ & $0,015-0,06$ & $0,5-2,0$ \\
\hline
\end{tabular}

\footnotetext{
a Datos en $\mathrm{mg} / \mathrm{L}$.

${ }^{b}$ Anfotericina.

c Fluorocitosina.

d Fluconazol.

e Itraconazol.

† Voriconazol.

9 Ketoconazol.

h Caspofungina.

American Type Culture Collection.
} 
CUADRO 2. Intervalos utilizados para validar los resultados obtenidos por el método del CLSI. ${ }^{\mathrm{a}}$

\begin{tabular}{llrrrrrrr}
\hline Cepa & \multicolumn{1}{c}{ Identificación } & ANB $^{\mathrm{b}}$ & 5-FC & FLC $^{\mathrm{d}}$ & ITC $^{\mathrm{e}}$ & VRC $^{f}$ & KTCg $^{\text {CPF }^{\mathrm{h}}}$ \\
\hline Cepa A, & Candida krusei ATCC 6258 & $0,50-2,0$ & $4,0-16,0$ & $16,0-\geq 64,0$ & $0,25-1,0$ & $0,12-1,0$ & $0,25-1,0$ & $0,25-1,0$ \\
Cepa B & Candida albicans ATCC 64548 & $0,12-0,5$ & $0,12-0,5$ & $0,25-1,0$ & $0,015-0,06$ & $0,015-0,06$ & $0,015-0,06$ & $0,03-0,12$ \\
Cepa C & Candida albicans ATCC 64550 & $0,25-1,0$ & $0,5-4,0$ & $8,0-\geq 64,0$ & $0,25-2,0$ & $0,25-1,0$ & $0,12-0,50$ & $0,03-0,12$ \\
Cepa D & Candida tropicalis ATCC 200956 & $4,0-8,0$ & $0,25-1,0$ & $32,0-\geq 64,0$ & $4,0-\geq 8,0$ & $\geq 8,0$ & $4,0-\geq 8,0$ & $0,03-0,25$ \\
Cepa E & Candida glabrata ATCC 90030 & $0,25-1,0$ & $0,25-1,0$ & $32,0-\geq 64,0$ & $0,12-1,0$ & $0,25-2,0$ & $2,0-\geq 8,0$ & $0,12-1,0$ \\
Cepa F & Saccharomyces cerevisiae ATCC 9763 & $0,25-1,0$ & $0,25-1,0$ & $2,0-8,0$ & $0,12-0,5$ & $0,03-0,25$ & $0,03-0,12$ & $0,25-1,0$ \\
Cepa G & Candida lusitaniae ATCC 200951 & $0,5-2,0$ & $0,25-1,0$ & $0,12-1,0$ & $0,015-0,06$ & $0,015-0,06$ & $0,015-0,06$ & $0,5-4,0$ \\
Cepa H & Candida parapsilosis ATCC 22019 & $0,50-2,0$ & $0,12-0,5$ & $0,5-4,0$ & $0,12-0,50$ & $0,03-0,25$ & $0,06-0,25$ & $0,5-4,0$ \\
\hline
\end{tabular}

a Datos en $\mathrm{mg} / \mathrm{L}$.

${ }^{\mathrm{b}}$ Anfotericina.

c Fluorocitosina.

d Fluconazol.

e Itraconazol.

† Voriconazol.

9 Ketoconazol.

${ }^{\text {h }}$ Caspofungina.

i American Type Culture Collection.

CUADRO 3. Resultados de la evaluación externa del desempeño. Laboratorios nacionales de referencia de Argentina, Brasil y Colombia, 2006

\begin{tabular}{|c|c|c|c|c|c|}
\hline $\begin{array}{l}\text { Laboratorio } \\
\text { nacional de } \\
\text { referencia }\end{array}$ & $\begin{array}{l}\text { Total de } \\
\text { CMls } \\
\text { realizadas }\end{array}$ & $\begin{array}{l}\text { CMls dentro } \\
\text { del intervalo } \\
\text { control }^{\mathrm{a}}\end{array}$ & $\begin{array}{l}\text { Porcentaje de } \\
\text { concordancias }\end{array}$ & $\begin{array}{l}\text { Discrepancias } \\
\text { mayores }^{b}\end{array}$ & $\begin{array}{c}\text { Porcentaje de } \\
\text { discrepancias mayores }\end{array}$ \\
\hline Argentina & 48 & 42 & 87,5 & 2 & 4,1 \\
\hline Brasil & 112 & 100 & 89,3 & 4 & 3,6 \\
\hline Colombia & 128 & 113 & 88,3 & 5 & 3,9 \\
\hline Total & 288 & 255 & 88,5 & 11 & 3,8 \\
\hline
\end{tabular}

a Número de CMls que notificaron los laboratorios sub-regionales dentro del intervalo de control.

b Se definieron las discrepancias mayores como aquellas CMls que se alejaban más de dos diluciones dobles del intervalo de control.

(CMI) notificadas por cada laboratorio que se incluía dentro del intervalo de control de calidad establecido para cada una de las cepas control (cuadros 1 y 2) (30, 32). Este intervalo es diferente según el método de referencia (EUCAST y CLSI) utilizado. En el caso de los valores fuera del intervalo control, se definieron discrepancias sustanciales o mayores como aquellas CMI mayores de dos diluciones dobles del intervalo de control. Los resultados de cada laboratorio fueron validados cuando se obtuvieron porcentajes de concordancia por encina de $85 \%$ y porcentajes de discrepancias sustanciales o mayores por debajo de $5 \%$.

Todos los cálculos se hicieron con el paquete informático Statistical Package for the Social Sciences (version 14.0; SPSS, S.L., Madrid, España).

En el cuadro 3 se presentan los porcentajes de CMI incluidos dentro de los intervalos de control establecidos, así como el número de discrepancias, para cada uno de los laboratorios participantes. Los tres laboratorios obtuvieron porcentajes de concordancia entre la CMI superiores a $85 \%$, con un por- centaje medio de $88,5 \%$, así como un porcentaje de discrepancias mayores por debajo de $5 \%$. Además, todos los laboratorios participantes en el ejercicio fueron capaces de detectar las cepas resistentes a fluconazol, así como la cepa multi-resistente Candida tropicalis ATCC200956.

$\mathrm{Al}$ analizar los resultados por antifúngicos, se observaron porcentajes de concordancia superiores a $90 \%$ con las CMI de anfotericina B y fluconazol. En el caso de itraconazol y voriconazol, esos porcentajes fueron de $85,2 \%$ y $86 \%$, respectivamente. Las discrepancias mayores fueron inferiores a $5 \%$ para todos los antifúngicos analizados.

\section{PROYECCIONES}

La OPS se ha abocado a la implementación de una iniciativa para reorientar las redes nacionales de laboratorios de la Región hacia un mayor entendimiento y cumplimiento de su papel en la vigilancia de la salud. La Red Regional de Vigilancia de las 
Infecciones Fúngicas Invasoras y Susceptibilidad a los Antifúngicos se creó en 2006, con los propósitos citados anteriormente. Toda organización de este tipo debe contar con procedimientos que permitan garantizar la calidad de sus resultados, y esa fue la razón que llevó a organizar el ejercicio de evaluación externa del desempeño, que además se utilizó como trabajo multicéntrico inaugural de la Red.

El ejercicio se efectuó para validar los procedimientos de detección de resistencia a los antifúngicos de los laboratorios de la Red. Estos procedimientos son esenciales para iniciar la vigilancia epidemiológica, ya que en sus hallazgos deben basarse las estrategias de control.

Los resultados del ejercicio pueden considerarse excelentes. Todos los laboratorios superaron los criterios de validación elegidos y detectaron las resistencias a anfotericina B y a los azoles. Estos datos permiten evaluar positivamente el desempeño de todos los laboratorios de la Red que participaron en el ejercicio, por lo que puede asegurarse que ha culminado el proceso de transferencia tecnológica y que los resultados de la vigilancia epidemiológica serán confiables para diseñar programas y estrategias de acción.

La Red debe ampliarse paulatinamente y mejorar su funcionamiento para adecuarse a los objetivos planteados. En 2007 se ha realizado un nuevo curso teórico/práctico que ha permitido consolidar la capacidad técnica de los dos centros de referencia sub-regional en Argentina y Brasil, así como ampliar la Red a nuevos países como Venezuela. Además, ya están en marcha nuevos ejercicios de evaluación, así como de intercambio de información y de pasantes, que servirán para analizar la capacidad de identificación y caracterización de las especies fúngicas. Asimismo, se continuará con las actividades formativas y de capacitación de profesionales y con el desarrollo de proyectos de investigación e innovación.
El objetivo de largo plazo de la Red consiste en transferir la tecnología para fortalecer la capacidad nacional y dar más autonomía a los países en la resolución concertada de los problemas.

\section{SUMMARY}

\section{Regional Laboratory Network for Surveillance of Invasive Fungal Infections and Antifungal Susceptibility in Latin America}

This article describes the general objectives of the Regional Laboratory Network for Surveillance of Invasive Fungal Infections and Antifungal Susceptibility in Latin America. Formation of the Network was coordinated by the Essential Medicines, Vaccines, and Health Technologies Unit of the Pan American Health Organization, with the technical and financial support of the National Center for Microbiology of the Carlos III Health Institute (Spain), and the technical support of the Microbiology Department of the Dr. C. Malbrán National Institute on Infectious Diseases (Argentina) and the Microbiology Unit of the Parasitology Service of the Adolfo Lutz Institute (Brazil).

The Network's principle objectives are epidemiological surveillance of invasive fungal infections through detection of antifungal resistance and identification of emergent, invasive fungal infections; establishment of norms and common protocols for early diagnosis of mycoses; and strengthening coordination, communications, and transference mechanisms among countries. The Network must be gradually implemented and must include staff training, a systematic process for sharing technology, evaluation of diagnostic techniques, identification of fungal species, and standardized tests for antifungal susceptibility.

Key words: mycoses, surveillance, diagnosis, antifungal agents, Latin America, Pan American Health Organization.

\section{REFERENCIAS}

1. Cuenca-Estrella M, Rodero L, GarciaEffron G, Rodriguez-Tudela JL. Antifungal susceptibilities of Candida spp. isolated from blood in Spain and Argentina, 1996-1999. J Antimicrob Chemother. 2002;49(6):981-7.

2. Cuenca-Estrella M, Gomez-Lopez A, Mellado E, Garcia-Effron G, RodriguezTudela JL. In vitro activities of ravuconazole and four other antifungal agents against fluconazole-resistant or -susceptible clinical yeast isolates. Anti- microb Agents Chemother. 2004;48(8): 3107-11.

3. Pfaller MA, Diekema DJ. Epidemiology of invasive candidiasis: a persistent public health problem. Clin Microbiol Rev. 2007;20(1):133-63.

4. Hazen KC. New and emerging yeast pathogens. Clin Microbiol Rev. 1995; 8(4):462-78.

5. Perfect JR, Schell WA. The new fungal opportunists are coming. Clin Infect Dis. 1996;22 (suppl 2):S112-8.
6. Denning DW. Invasive aspergillosis. Clin Infect Dis. 1998;26(4):781-803.

7. Walsh TJ, Groll AH. Emerging fungal pathogens: evolving challenges to immunocompromised patients for the twenty-first century. Transpl Infect Dis. 1999;1(4):247-61.

8. Latge JP. Aspergillus fumigatus and aspergillosis. Clin Microbiol Rev. 1999; 12(2):310-50.

9. Singh N. Trends in the epidemiology of opportunistic fungal infections: predis- 
posing factors and the impact of antimicrobial use practices. Clin Infect Dis. 2001;33(10):1692-6.

10. Singh N. Invasive aspergillosis in organ transplant recipients: new issues in epidemiologic characteristics, diagnosis, and management. Medical Mycology. 2005;43(suppl 1):S267-70.

11. Almirante $\mathrm{B}$, Rodriguez D, Park BJ Cuenca-Estrella M, Planes AM, Almela $\mathrm{M}$, et al. Epidemiology and predictors of mortality in cases of Candida bloodstream infection: results from populationbased surveillance, Barcelona, Spain, from 2002 to 2003. J Clin Microbiol. 2005; 43(4):1829-35.

12. Munoz P, Bouza E, Cuenca-Estrella M, Eiros JM, Perez MJ, Sanchez-Somolinos $\mathrm{M}$, et al. Saccharomyces cerevisiae fungemia: an emerging infectious disease. Clin Infect Dis. 2005;40(11):1625-34.

13. Guarro J, Kantarcioglu AS, Horre R, Rodriguez-Tudela JL, Cuenca EM, Berenguer J, et al. Scedosporium apiospermum: changing clinical spectrum of a therapy-refractory opportunist. Med Mycol. 2006;44(4):295-327.

14. González A, Tobón AM. Infecciones micóticas oportunistas en pacientes con VIH/SIDA. Infectio. 2006;10(4): 279-88.

15. Benson CA, Kaplan JE, Masur H, Pau A, Holmes KK, CDC, et al. Treating opportunistic infections among HIV-infected adults and adolescents: recommendations from CDC, the National Institutes of Health, and the HIV Medicine Association/Infectious Diseases Society of America. MMWR. 2004;53(RR-15): 1-112.

16. Martin GS, Mannino DM, Eaton S, Moss $M$. The epidemiology of sepsis in the United States from 1979 through 2000 N Engl J Med. 2003;348(16):1546-54.

17. Gavalda J, Len O, San Juan R, Aguado JM, Fortun J, Lumbreras C, et al. Risk factors for invasive aspergillosis in solid-organ transplant recipients: a case-control study. Clin Infect Dis. 2005; 41(1): 52-9.
18. Arendrup MC, Fuursted K, GahrnHansen B, Jensen IM, Knudsen JD, Lundgren $B$, et al. Seminational surveillance of fungemia in Denmark: notably high rates of fungemia and numbers of isolates with reduced azole susceptibility. J Clin Microbiol. 2005;43(9):4434-40.

19. Morgan J, Meltzer MI, Plikaytis BD, Sofair AN, Huie-White S, Wilcox S, et al. Excess mortality, hospital stay, and cost due to candidemia: a case-control study using data from population-based candidemia surveillance. Infect Control Hosp Epidemiol. 2005;26(6):540-7.

20. Pinner RW, Rebmann CA, Schuchat A Hughes JM. Disease surveillance and the academic, clinical, and public health communities. Emerg Infect Dis. 2003; 9(7):781-7.

21. Shetty SS, Harrison LH, Hajieh RA, Taylor T, Mirza SA, Schmidt AB, et al. Determining risk factors for candidemia among newborn infants from population-based surveillance: Baltimore, Maryland, 19982000. Pediatr Infect Dis J. 2005;24(7):601-4.

22. Grigull L, Beier R, Schrauder A, Kirschner P, Loening L, Jack T, et al. Invasive fungal infections are responsible for one-fifth of the infectious deaths in children with ALL. Mycoses. 2003;46 (11-12):441-6

23. Nucci M, Marr KA, Queiroz-Telles $F$, Martins CA, Trabasso P, Costa S, et al. Fusarium infection in hematopoietic stem cell transplant recipients. Clin Infect Dis. 2004; 38(9):1237-42.

24. Castagnola E, Cesaro S, Giacchino M, Livadiotti S, Tucci F, Zanazzo G, et al. Fungal infections in children with cancer: a prospective, multicenter surveillance study. Pediatr Infect Dis J. 2006; 25(7):634-9.

25. Cuenca-Estrella M, Rodriguez D, Almirante $\mathrm{B}$, Morgan J, Planes AM, Almela $\mathrm{M}$, et al. In vitro susceptibilities of bloodstream isolates of Candida species to six antifungal agents: results from a population-based active surveillance programme, Barcelona, Spain, 2002 2003. J Antimicrob Chemother. 2005; 55(2):194-9.

26. Cuenca-Estrella $M$, Rodriguez-Tudela JL. Should antifungal treatments be based upon results of antifungal susceptibility testing? Rev Iberoam Micol. 2002;19(3):133-8.

27. Mellado E, Cuenca-Estrella $M$ Rodriguez-Tudela JL. Clinical relevance of mechanisms of antifungal drug resistance in filamentous fungi. Enferm Infecc Microbiol Clin. 2002;20(10):523-9.

28. White TC. Mechanism of resistance to antifungal agents. En: Pfaller MA, ed. Manual of Clinical Microbiology. Washington: ASM Press, 2003. Pp. 1869-79.

29. Clinical Laboratory Standard Institute. Reference Method for Broth Dilution Antifungal Susceptibility Testing of Yeast; Approved Standard-Second Edition. Document M27-A2. Wayne, Pennsylvania, USA; 2002.

30. Cuenca-Estrella M, Moore CB, Barchiesi F, Bille J, Chryssanthou E, Denning DW, et al. Multicenter evaluation of the reproducibility of the proposed antifungal susceptibility testing method for fermentative yeasts of the Antifungal Susceptibility Testing Subcommittee of the European Committee on Antimicrobial Susceptibility Testing (AFST-EUCAST). Clin Microbiol Infect. 2003;9(6):467-74.

31. Clinical Laboratory Standards Institute. Method for disk diffusion susceptibility testing of yeasts. Approved guideline. Document M44-A. Wayne, Pennsylvania, USA; 2004

32. Barry AL, Pfaller MA, Brown SD, Espinel-Ingroff A, Ghannoum MA, Knapp C, et al. Quality control limits for broth microdilution susceptibility tests of ten antifungal agents. J Clin Microbiol. 2000;38(9):3457-9. 